\title{
In-Out-In: The Use of a New Sutureless Endovascular Bypasses Technique as an Alternative to Treat High-risk Surgical Patients with Extensive Femoropopliteal Lesion
}

Roberto Teodoro Beck ${ }^{1}$, Milton Sérgio Bohatch Júnior ${ }^{2 *}$, Marcelo Haddad Dantas ${ }^{1}$, Amanda Fernandes Vidal da Silva ${ }^{3}$, Camila Perli Pinto ${ }^{4}$

${ }^{1}$ Institute of Angiology Vascular and Endovascular Surgery, Joinville, Santa Catarina, Brazil; ${ }^{2}$ Department of Vascular Surgery, São Paulo University, Ribeirão Preto, São Paulo, Brazil; ${ }^{3}$ São José Municipal Hospital, Joinville, Santa Catarina, Brazil; ${ }^{4}$ Universidade Do Oeste Paulista, Western São Paulo University, Presidente Prudente, São Paulo, Brazi

\begin{abstract}
Sutureless Viabahn anastomosis has been used as a promising technique in selected cases. The current study conducted a sutureless femoropopliteal bypass, through percutaneous accesses and an extra-anatomical trajectory, in a patient with critical limb ischemia. This technique, which is minimally invasive, has become a femoropopliteal revascularization alternative to be applied to high-risk patients with extensive occlusions.
\end{abstract}

Keywords: Endovascular; Bypasses technique; Femoropopliteal lesion; Vascular

\section{INTRODUCTION}

Critical limb ischemia (CLI) is defined as a persistent ischemic pain, even at rest, and as the incidence of ulcer or gangrene for longer than two weeks [1-3]. CLI is the most severe type of peripheral arterial vascular disease; its annual incidence in Europe and in the United States reaches 500 to 1000 cases per million individuals $[4,5]$.

Revascularization (endovascular or conventional surgery) is gold standard and it is used to prevent amputations. The surgical decision must be individualized, since there is no well-defined consensus about it among health services, despite the current tendency towards endovascular therapy [6]. Many patients are not suitable candidates for any revascularization procedure due to comorbidities and/or complex arterial lesions, a fact that generates therapeutic challenges and requires constant innovations to avoid major amputations [6].

The current study describes the use of an unprecedented sutureless endovascular bypass technique as an alternative to treat a high-risk surgical patient with critical ischemia and extensive arterial lesion (TASC D).

\section{CASE REPORT}

A 60-year-old male patient presenting COPD, diabetes mellitus, chronic kidney disease in dialytic treatment, congestive heart failure with myocardial revascularization (10 years before), and peripheral arterial occlusive disease with endovascular correction of thoracoabdominal aneurysm (conducted in October 2017) was admitted at the emergency room, in March 2018, with pain at rest and toe necrosis in progress after 18 days. His vital signs were stable upon examination. His left foot had cyanosis and infected necrosis from the $3^{\text {rd }}$ to the $5^{\text {th }}$ toe. Vascular examination of the affected limb has shown pulse only in the femoral artery, monophasic flow in the popliteal and anterior tibial arteries, and ankle-brachial index (ABI) of $35 \mathrm{mmHg}$. Doppler ultrasound examination presented femoropopliteal obstruction showing rehabilitation due to collateral circulation above the patella, as well as posterior tibial artery occlusion. The patient underwent left lower limb arteriography with contralateral puncture, which showed superficial femoral artery occlusion (at the origin of the artery), extensive calcification, suprapatellar popliteal artery rehabilitation, and satisfactory drainage through the anterior fibular and tibial arteries (TASC D classification). Anterograde recanalization attempt was thwarted. Given the lack of autologous graft associated with high surgical risk (Lee Vasc scores 10), a sutureless endovascular bypass was performed, as described in the stages below:

\section{Stage 1}

After the diagnostic arteriography described above, a radioscopy-

${ }^{*}$ Correspondence to: Milton Sérgio Bohatch Júnior, Vascular Surgery Resident, Department of Vascular Surgery, São Paulo University, Ribeirão Preto, São Paulo, Brazil, Tel: 4799433299; E-mail: milton.jr87@hotmail.com

Received: November 24, 2019; Accepted: January 02, 2020; Published: January 11, 2020

Citation: Beck RT, Bohatch Júnior MS, Dantas MH, Vidal da Silva AF, Perli Pinto C (2020) IN-OUT-IN: The use of a new Sutureless Endovascular Bypasses Technique as an Alternative to treat High-risk Surgical Patients with Extensive Femoropopliteal Lesion. J Vasc Med Surg 8:1. doi: 10.35248/2329-6925.20.8.385.

Copyright: $@ 2019$ Beck RT, et al. This is an open-access article distributed under the terms of the Creative Commons Attribution License, which permits unrestricted use, distribution, and reproduction in any medium, provided the original author and source are credited. 
guided retrograde access was performed to pass an $8 \mathrm{Fr}$ introducer at the origin of the superficial femoral artery (SFA) by using the parietal calcification as reference (Figure 1A). Figure 1B shows the retrograde access reaching the true lumen.

\section{Stage 2}

An ipsilateral anterograde access was performed to pass an $8 \mathrm{Fr}$ introducer in the Common Femoral Artery (CFA). Subsequently, a $0.035 \mathrm{~mm}$ hydrophilic guidewire was passed through the introducers to connect the two accesses, similar to the clothesline technique. The introducers were removed to allow passing a long Balkin guiding sheath $(45 \mathrm{~cm}, 8 \mathrm{Fr}$; COOK MEDICAL LLC, Indiana, USA), which was introduced just above the inguinal ligament and exteriorized near the middle third of the thigh.

\section{Stage 3}

Radioscopy-guided anterograde access was performed in the popliteal artery (PA) above the patella. In order to do so, partial knee flexion and hip abduction were performed to allow arterial access through the medial aspect of the thigh near its middle third. After the access to the true lumen was confirmed through contrast infusion, an $8 \mathrm{Fr}$ introducer was introduced and a $0.035 \mathrm{~mm}$ STIFF hydrophilic guidewire was inserted until it reached the leg vessels. Subsequently, the introducer inserted in the PA was removed from it and the guidewire was passed again through the long sheath until it reached the inguinal region. Thus, the 3 accesses were connected to enable the long sheath to progress from the CFA to the PA, in a path that was externalized in the middle third of the thigh (In-Out-In Path). A small incision (approximately $2 \mathrm{~cm}$ ) was made between sheath externalizations to allow the sheath to be buried in the subcutaneous tissue. Figure $1 \mathrm{C}-1 \mathrm{E}$ shows the path of the sheath connecting the 3 accesses.

\section{Stage 4}

Finally, 4 Viabahn endoprostheses were released $(7 \times 150 \mathrm{~mm}+7$ x $150 \mathrm{~mm}+7$ x $100 \mathrm{~mm}+8$ x 50 mm; WL Gore, Flagstaff, AZ, USA) Figure 2; they were all connected through telescoping, always with approximately $4 \mathrm{~cm}$ overlap from the PA to the CFA (Figure 3A-3C). Residual stenoses were subjected to balloon angioplasty $(7 \times 100 \mathrm{~mm})$ and presented excellent results (Figure 3D-3E). Control arteriography showing bypass perviousness can be seen in Figure 4. The tomographic imaging shown in Figure 5 depicts the in-out-in path of the bypass along the lower limb.

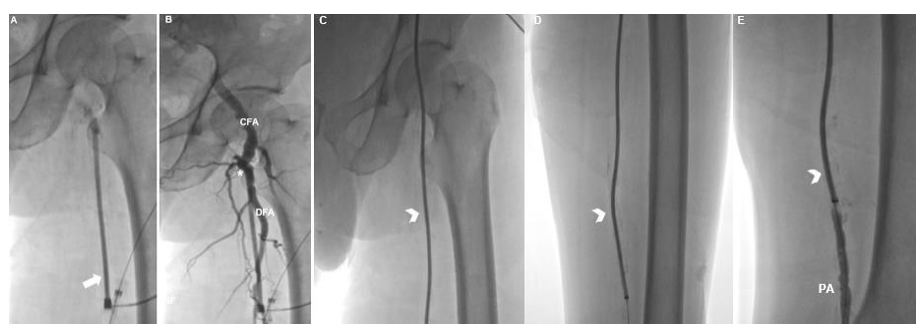

Figure 1: Sequences of accesses and passing of the long Balkin sheath (45 $\mathrm{cm})$. A: Retrograde access to the superficial femoral artery; B: Arteriography confirming access to true lumen (B), C/D: Anterograde access to the common femoral artery in the inguinal region, based on the insertion of the $45 \mathrm{~cm}$-long Balkin sheath connecting the 3 accesses. E: Arteriography confirming the access to the true lumen of the popliteal artery. CFA: Common femoral artery; DFA: Deep femoral artery; PA: Popliteal artery; $\left.{ }^{*}\right)$ superficial femoral artery occlusion; arrow: 8 Fr introducer; Arrowhead: $45 \mathrm{~cm}$-long Balkin sheath.

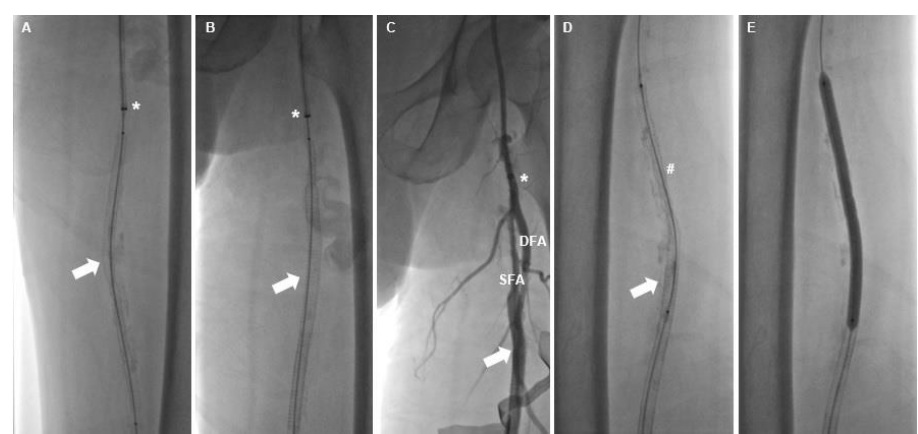

Figure 2: A: The Viabahn stent was released (arrow) in the distal third of the thigh. B: The Viaban stent was released (arrow) in the middle third of the thigh. C: The angiography of the limb showed flow from the common femoral artery on. D: The Viabahn stent showed stenosis areas. E: Correction of stenosis areas through angioplasty. ${ }^{*}$ ) Arrow: Viabahn stent. SFA: superficial femoral artery; DFA: deep femoral artery; $(\#)$ : stenosis. $\left(^{*}\right)$ : Balkin sheath.
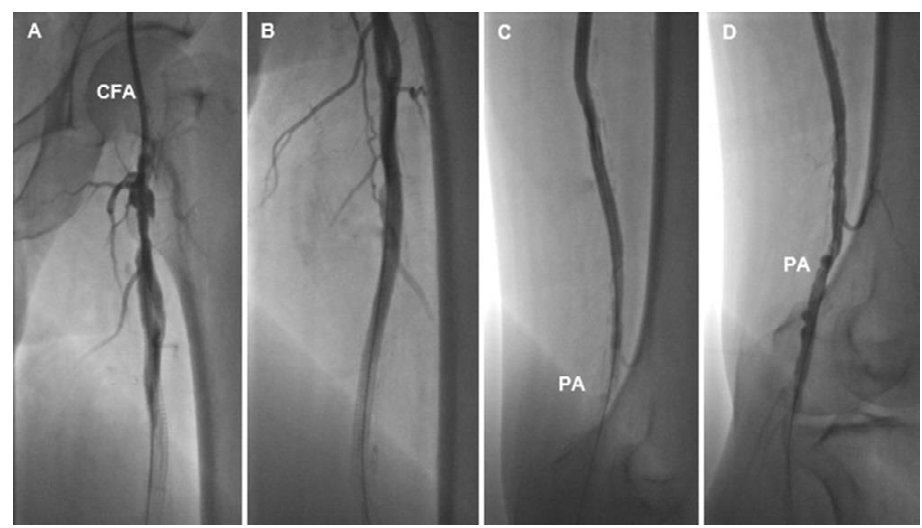

Figure 3: Control angiography: A: Proximal third of the thigh, B: Middle third of the thigh, C and D: Distal third of the thigh, CFA: Common Femoral Artery; SFA: Superficial Femoral Artery; PA: Popliteal Artery.

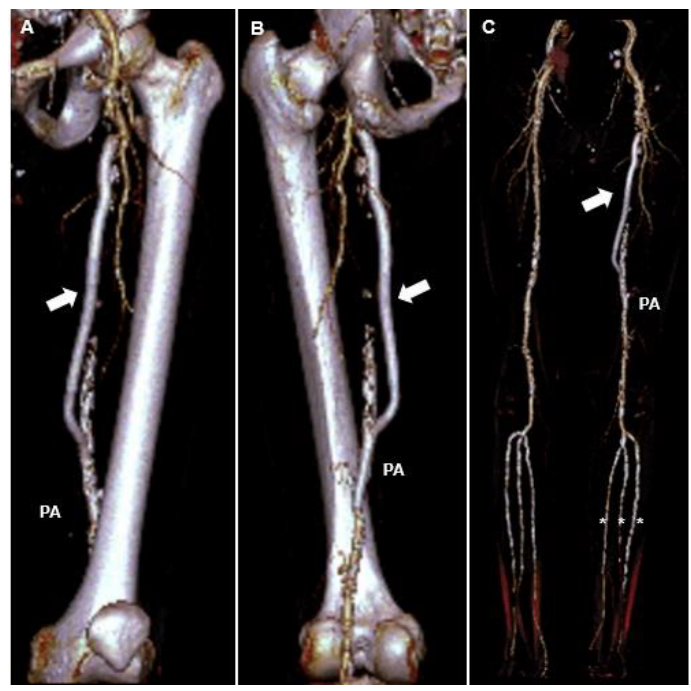

Figure 4: Angiotomography of the lower limbs with 3D reconstruction, A: Anterior View of the Bypass (arrow), B: Posterior view of the bypass (arrow), C: The bypass is displayed to demonstrate the permeability by filling the popliteal artery (PA) and the leg vessels $\left({ }^{*}\right)$.

The patient presented anterior tibial pulse, limb warming and pain involution. At the 14th postoperative day, after the necrotic area was defined, he was subjected to full amputation of the 5th toe and to partial amputation of the 4th and 3rd toes. This procedure was followed by seven sessions of hyperbaric oxygen therapy. He was discharged 30 days after surgery. At the 6-month evaluation, the patient did not have symptoms of intermittent claudication 


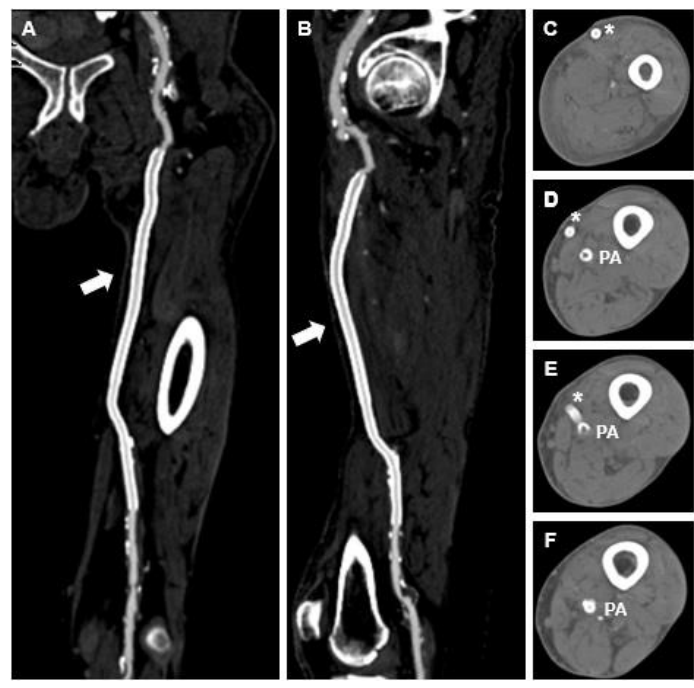

Figure 5: Angiotomography of the lower limb showing bypass trajectory (arrow) in the subcutaneous tissue almost along the entire extension of the thigh. A: Coronal cross-section. B: Sagittal cross-section. C-F: axial crosssections. Arrow: bypass with Viabahn stent; $\left(^{*}\right)$ bypass with Viabahn stent in extra anatomical path; PA: Popliteal Artery.

or trophic lesions; he maintained distal pulse and the vascular Doppler examination showed flow in the bypass topography, triphasic flow in the anterior tibial artery and ABI of 0.98.

The presentation of this case for scientific dissemination was authorized by the patient.

\section{DISCUSSION}

The standard technique focused on anastomosis requires extensive vessel exposure, circumferential dissection and occlusion [7]. Intraoperative damage in the arterial wall is a well-known contributing factor to intimal hyperplasia, anastomotic stenosis and graft failure [8]. On the other hand, sutureless telescoping anastomosis, which was originally described as VORTEC (Viabahn Open Rebranching Technique) [7], enables revascularization based on arterial puncture (only) with minimal vessel manipulation. This hybrid revascularization technique enabled significant advancements in the treatment of thoracoabdominal and parental aortic aneurysms $[9,10]$. The Viabahn stent graft (WL Gore \& Associates, Flagstaff, Ariz) is composed of polytetrafluoroethylene attached to a flexible nitinol stent, which was initially developed to treat iliofemoral occlusive disease [7]. The current study has only used percutaneous access and all anastomoses were performed through sutureless telescoping. Thus, the herein performed bypass required minimal arterial manipulation and, consequently, it caused less surgical stress.

Innovations in surgical techniques are a remarkable feature of endovascular therapy, which enables the recovery of limbs that, otherwise, would be considered untreatable.

\section{CONCLUSION}

The present study described a new endovascular technique-called In-Out-In-used to treat femoropopliteal lesions based on the combination between sutureless vascular access and anastomosis techniques. Such combination enables restoring the blood flow between distant vessels and it achieves satisfactory results in highrisk surgical patients affected by critical limb ischemia disease with extensive arterial lesion (TASC D).

\section{CONFLICT OF INTERESTS}

The authors declare no conflict of interests.

\section{REFERENCES}

1. Abu Dabrh AM, Steffen MW, Undavalli C, Asi N, Wang Z, Elamin $\mathrm{MB}$, et al. The natural history of untreated severe or critical limb ischemia. J Vasc Surg. 2015;62(6):1642-1651.

2. Stoner MC, Calligaro KD, Chaer RA, Dietzek AM, Farber A, Guzman RJ, et al. Reporting standards of the Society for Vascular Surgery for endovascular treatment of chronic lower extremity peripheral artery disease. J Vasc Surg. 2016;64(1):1-21.

3. Norgren L, Patel MR, Hiatt WR, Wojdyla DM, Fowkes FGR, Baumgartner I, et al. Outcomes of Patients with Critical Limb Ischaemia in the EUCLID Trial. Eur J Vasc Endovasc Surg. 2018;55(1):109-117.

4. Teraa M, Conte MS, Moll FL, Verhaar MC. Critical Limb Ischemia: Current Trends and Future Directions. J Am Heart Assoc. 2016;5(2):1-8.

5. Kinlay S. Management of Critical Limb Ischemia. Circ Cardiovasc Interv. 2016;9(2):1-23.

6. Dosluoglu HH, Lall P, Harris LM, Dryjski ML. Long-term limb salvage and survival after endovascular and open revascularization for critical limb ischemia after adoption of endovascular-first approach by vascular surgeons. J Vasc Surg. 2012;56(2):361-371.

7. Pfammatter T, Rancic Z, Veith FJ, Genoni M, Mayer D, Lachat M, et al. New Technique to Facilitate Renal Revascularization with Use of Telescoping Self-Expanding Stent Grafts: VORTEC. Vascular. 2008;16(2):69-72.

8. Ginzburg V, Leytzin A, Szendro G, Greenberg G, Mayzler O. Use of ViaBahn Open Revascularisation Technique for Above-knee Femoropopliteal Anastomosis: A Technical Note. Eur J Vasc Endovasc Surg. 2011;42(2):202-205.

9. Narayanan S, Shalhoub J, Karunanithy N, Burfitt N. Primary sutureanastomosis of the Viabahn endoprosthesis to a native artery. J Vasc Surg. 2010;51(5):1297-1299.

10.Donas KP, Rancic Z, Lachat M, Pfammatter T, Frauenfelder T, Veith FJ, et al. Novel sutureless telescoping anastomosis revascularization technique of supra-aortic vessels to simplify combined open endovascular procedures in the treatment of aortic arch pathologies. J Vasc Surg. 2010;51(4):836-841. 